\title{
A Survey of Peri-Colonoscopy Management of Anti-Diabetic Medications
}

\author{
Byeori Lee ${ }^{1^{*}}$, Deepanshu Jain ${ }^{2^{*}}$ and Michael Rajala ${ }^{2}$ \\ ${ }^{1}$ Department of Internal Medicine, ${ }^{2}$ Division of Gastroenterology, Department of Digestive Diseases and Transplantation, Albert Einstein \\ Healthcare Network, Philadelphia, PA, USA
}

Over 14 million colonoscopies are performed annually in the United States. ${ }^{1}$ The most well-recognized indication for colonoscopy is colorectal cancer (CRC) screening and surveillance. In addition, colonoscopy is widely used for the diagnostic examinations of symptoms (abdominal pain, rectal bleeding, or changes in bowel habits) and positive results of other CRC screening tests (positive fecal occult blood). Diabetes mellitus (DM) is among the most common illnesses in the United States. According to the most recent statistics, 23.1 million people or $7.2 \%$ of the United States population have been diagnosed with DM, with 95\% of them being diagnosed with type $2 \mathrm{DM}^{2}$ The prevalence of DM has increased over the past decades. ${ }^{3}$ The percentage of adults with DM increased with age, reaching $12.7 \%$ among those aged 45-64 years and $20.8 \%$ among those aged 65 years or older. In other words, approximately $10 \%-20 \%$ of patients undergoing colonoscopy may have diabetes.

Adequate pre-procedure bowel preparation is key to the success of colonoscopy because poor preparation results in longer procedure times, lower detection rates of lesions, and a higher risk of complications. ${ }^{4,5}$ Since bowel preparations and

Received: January 1, 2020 Revised: March 21, 2020

Accepted: March 27, 2020

Correspondence: Byeori Lee

Department of Internal Medicine, Albert Einstein Healthcare Network, 5501 Old

York Road, Philadelphia, PA 19141, USA

Tel: +1-215-456-8210, Fax: +1-215-455-1933, E-mail: leebyeor@einstein.edu

ORCID: https://orcid.org/0000-0001-9971-5345

*These authors contributed equally to this study.

cc This is an Open Access article distributed under the terms of the Creative Commons Attribution Non-Commercial License (http://creativecommons.org/ licenses/by-nc/3.0) which permits unrestricted non-commercial use, distribution, and reproduction in any medium, provided the original work is properly cited. restrictions in diet for colonoscopy can affect blood glucose levels in diabetic patients, it is important to adjust anti-diabetic medications for the planned procedure. Unfortunately, there is no guideline for this adjustment, and different centers (and individual practitioners) have their self-made regimens based on the general principles of action of these medications. Not infrequently, diabetic patients present with hypoglycemia or hyperglycemia on the day of the procedure, which leads to a delay in starting the procedure on time. ${ }^{6}$ Although this may be attributed to multiple factors such as instructions being too complex, patients forgetting verbal instructions over time (longer intervals between outpatient appointment and procedure day), or patients having poor control of their diabetes at baseline, we hypothesize that the lack of evidence-based recommendations leads to a lack of consensus which causes confusion and mismanagement at the patient level.

Through our research, we aimed to find real-world practices of gastroenterology providers in the management of anti-diabetic medications during the peri-colonoscopy period for type 2 DM patients.

We created a 20-question online survey using Google Survey to gather data on real-world practices of gastroenterology providers for the management of diabetes using anti-diabetic medications during the peri-colonoscopy period for type 2 DM patients. The first question of the survey established the title of the responder (attending physician, fellow-in-training, or nurse practitioner/physician assistant).

The rest of the questions were designed to ask anti-diabetic medication adjustments specific to six non-insulin medications and four insulin regimens for each meal on the day before and the day (morning) of colonoscopy. The six non-insulin medications included biguinades, sodium-glucose 
cotransporter-2 inhibitors, dipeptidyl peptidase 4 inhibitors, thiazolidinediones, sulfonylureas, and meglitinides. Four insulin regimens included long-acting, intermediate-acting, short-acting, and premixed insulins. Examples of common brand names were provided for each class of medications. For non-insulin medications, the responders were given options that included "do not take", "take", and "do not know". For insulin regiments, the options included "do not take", "decrease by half", "take the usual dose", and "do not know". Because bowel preparation time and methods can vary depending on the time of the colonoscopies, we clarified in our survey that all the colonoscopies would be in the morning in this scenario.

The online survey was distributed to thirty-three providers including attending physicians, fellow physicians, or nurse practitioners/physician assistants who are routinely involved in planning colonoscopies at our medical center (Einstein Medical Center, Philadelphia, PA, USA). The answers from each provider were collected after 4 weeks. For each question, the percentages of the answers were calculated. Two expert endocrinologists were consulted to give best practice recommendations regarding the same medications that were included in the questionnaires. The collected answers were compared with the endocrinologists' opinion. The percentages of the answers that were in agreement or disagreement with the endocrinologists' opinions were calculated.

Here is the actual Google Survey link:

https://docs.google.com/forms/d/e/1FAIpQLS f6ihaIO1GtwsZXdoGa1js5wZJ7411U7uiC90XeOM01JNM-NA/viewform?usp=sf_link

Among 33 providers, 24 providers (72.7\%) answered the survey. Of them, 12/24 (50\%) were attending physicians, 6/24 (25\%) were fellows-in-training, and 6/24 (25\%) were nurse practitioners/physician assistants. The calculated percentages and cumulative numbers for responses specific to each medication and meal are summarized in Tables 1,2, and 3. The answers were compared with the endocrinologists' opinion and the percentages in agreement and disagreement were calculated. The answers with the footnote represent the appropriate answers recommended by the endocrinologists.

Irrespective of indication, adequate pre-procedure bowel preparation is key to successful colonoscopy. Poor preparation results in longer procedure times, lower detection rates of lesions, and a higher risk of complications. ${ }^{4,5}$ Restrictions in diet the day before colonoscopy and fasting after midnight are an integral part of any bowel preparation regimen regardless of the laxative agent used. In addition, patients with type $2 \mathrm{DM}$ need recommendations pertinent to the management of their anti-diabetic medications in the peri-colonoscopy period. Unfortunately, no evidence-based guidelines exist. Even though there are several recommendations and guidelines available for perioperative management of anti-diabetic medications, those guidelines are not applicable to peri-colonoscopy management due to the unique nature of bowel preparation and dietary restrictions. ${ }^{7-9} \mathrm{~A}$ recent review by Hochberg et al. suggested instructions for antihyperglycemic medications precolonoscopy. ${ }^{6}$ The recommendations in this review are similar to the expert recommendations we used for our study but not exactly the same, which again highlights the need for actual data to accurately come up with evidence-based practice recommendations.

The aim of this study was to find real-world practices of gastroenterology providers in the management of anti-diabetic medications in the peri-colonoscopy period for type 2 DM patients. As presented in Tables 1,2 and 3, there is a great disparity amongst gastroenterology providers and also when compared to endocrinologist recommendations. For medi-

Table 1. Non-Insulin Medication Management on the Day before a Colonoscopy

\begin{tabular}{lcccc}
\hline Non-insulin medications & Time of day & Do not take & Take & Do not know \\
\hline Metformin & Morning & $29.2 \%(7 / 24)^{\mathrm{a}}$ & $70.8 \%(17 / 24)$ & $0.0 \%(0 / 24)$ \\
& Evening & $41.7 \%(10 / 24)^{\mathrm{a}}$ & $54.2 \%(13 / 24)$ & $4.2 \%(1 / 24)$ \\
SGLT inhibitor & Daily & $45.8 \%(11 / 24)^{\mathrm{a}}$ & $45.8 \%(11 / 24)$ & $8.3 \%(2 / 24)$ \\
DPP-4 inhibitor & Daily & $41.7 \%(10 / 24)$ & $54.2 \%(13 / 24)^{\mathrm{a})}$ & $4.2 \%(1 / 24)$ \\
Thiazolidinediones & Daily & $50.0 \%(12 / 24)$ & $45.8 \%(11 / 24)^{\mathrm{a})}$ & $4.2 \%(1 / 24)$ \\
Sulfonylureas & Daily & $58.3 \%(14 / 24)^{\mathrm{a})}$ & $41.7 \%(10 / 24)$ & $0.0 \%(0 / 24)$ \\
Meglitinide & Breakfast & $\left.41.7 \%(10 / 24)^{\mathrm{a}}\right)$ & $33.3 \%(8 / 24)$ & $25.0 \%(6 / 24)$ \\
& Lunch & $\left.45.8 \%(11 / 24)^{\mathrm{a}}\right)$ & $29.2 \%(7 / 24)$ & $25.0 \%(6 / 24)$ \\
& Dinner & $54.2 \%(13 / 24)^{\mathrm{a})}$ & $20.8 \%(5 / 24)$ & $25.0 \%(6 / 24)$ \\
\hline
\end{tabular}

DPP-4, dipeptidyl peptidase 4; SGLT, sodium-glucose cotransporter.

a) The appropriate answers recommended by the endocrinologists. 
Table 2. Insulin Regimen Management on the Day before a Colonoscopy

\begin{tabular}{lccccc}
\hline Insulin medications & Time of day & Do not take & Decrease by half & Take the usual dose & Do not know \\
\hline Long-acting Insulin & Bedtime & $0.0 \%(0 / 24)$ & $87.5 \%(21 / 24)$ & $8.3 \%(2 / 24)^{\mathrm{a})}$ & $4.2 \%(1 / 24)$ \\
Intermediate-acting Insulin & Breakfast & $0.0 \%(0 / 24)$ & $70.8 \%(17 / 24)$ & $25.0 \%(6 / 24)^{\mathrm{a})}$ & $4.2 \%(1 / 24)$ \\
& Dinner & $12.5 \%(3 / 24)$ & $62.5 \%(15 / 24)$ & $20.8 \%(5 / 24)^{\mathrm{a})}$ & $4.2 \%(1 / 24)$ \\
Short-acting Insulin & Breakfast & $25.0 \%(6 / 24)$ & $50.0 \%(12 / 24)^{\mathrm{a})}$ & $20.8 \%(5 / 24)$ & $4.2 \%(1 / 24)$ \\
& Lunch & $29.2 \%(7 / 24)$ & $41.7 \%(10 / 24)^{\mathrm{a})}$ & $25.0 \%(6 / 24)$ & $4.2 \%(1 / 24)$ \\
Premix Insulin & Dinner & $33.3 \%(8 / 24)$ & $41.7 \%(10 / 24)^{\mathrm{a})}$ & $20.8 \%(5 / 24)$ & $4.2 \%(1 / 24)$ \\
& Breakfast & $0.0 \%(0 / 24)$ & $70.8 \%(17 / 24)^{\mathrm{a})}$ & $25.0 \%(6 / 24)$ & $4.2 \%(1 / 24)$ \\
& Dinner & $12.5 \%(3 / 24)$ & $70.8 \%(3 / 24)^{\mathrm{a})}$ & $12.5 \%(3 / 24)$ & $4.2 \%(1 / 24)$ \\
\hline
\end{tabular}

a) The appropriate answers recommended by the endocrinologists.

Table 3. Non-Insulin Medication and Insulin Regimen Management in the Morning of a Colonoscopy

\begin{tabular}{|c|c|c|c|c|}
\hline Non-insulin medications & Do not take & \multicolumn{2}{|c|}{ Take } & Do not know \\
\hline Metformin & $66.7 \%(16 / 24)^{\mathrm{a})}$ & \multicolumn{2}{|c|}{$33.3 \%(8 / 24)$} & $0.0 \%(0 / 24)$ \\
\hline SGLT inhibitor & $79.2 \%(19 / 24)^{a)}$ & \multicolumn{2}{|c|}{$8.3 \%(2 / 24)$} & $12.5 \%(3 / 24)$ \\
\hline DPP-4 inhibitor & $75.0 \%(18 / 24)^{\mathrm{a})}$ & \multicolumn{2}{|c|}{$12.5 \%(3 / 24)$} & $12.5 \%(3 / 24)$ \\
\hline Thaliazolinediones & $87.5 \%(21 / 24)^{\mathrm{a})}$ & \multicolumn{2}{|c|}{$8.3 \%(2 / 24)$} & $4.2 \%(1 / 24)$ \\
\hline Sulfonylureas & $91.7 \%(22 / 24)^{\mathrm{a})}$ & \multicolumn{2}{|c|}{$4.2 \%(1 / 24)$} & $4.2 \%(1 / 24)$ \\
\hline Meglitinide & $87.5 \%(21 / 24)^{\mathrm{a})}$ & \multicolumn{2}{|c|}{$0.0 \%(0 / 24)$} & $12.5 \%(3 / 24)$ \\
\hline Insulin medications & Do not take & Decrease by half & Take the usual dose & Do not know \\
\hline Intermediate-acting Insulin & $62.5 \%(15 / 24)$ & $33.3 \%(8 / 24)^{\mathrm{a})}$ & $0.0 \%(0 / 24)$ & $4.2 \%(1 / 24)$ \\
\hline Short-acting Insulin & $87.5 \%(21 / 24)^{\mathrm{a})}$ & $8.3 \%(2 / 24)$ & $0.0 \%(0 / 24)$ & $4.2 \%(1 / 24)$ \\
\hline Premix Insulin & $70.8 \%(17 / 24)^{\mathrm{a})}$ & $25.0 \%(6 / 24)$ & $0.0 \%(0 / 24)$ & $4.2 \%(1 / 24)$ \\
\hline
\end{tabular}

DPP-4, dipeptidyl peptidase 4; SGLT, sodium-glucose cotransporter.

a) The appropriate answers recommended by the endocrinologists.

cations that are less familiar, there were more "do not know" answers suggesting the providers do not feel comfortable making decisions regarding these newer or uncommonly used medications. Unfortunately, for the most common medications like metformin and long-acting insulin, the majority was in disagreement with the endocrinologists' opinion. For metformin, most gastroenterology providers chose "take" as the answer, likely due to the well-established lack of associated hypoglycemia. However, the cessation of metformin within 48 hours before a surgery or procedure is recommended due to the potential risk of lactic acidosis sue to possible bleeding, dehydration, or contrast-induced acute kidney injury. ${ }^{9}$ During the colonoscopy, the expected bleeding risk is low and no contrast is used. However, due to bowel preparation, there is a risk of dehydration and electrolyte imbalance. For this reason, endocrinologists recommended stopping metformin the day before and morning of the colonoscopy. For patients who are fasting, gastroenterology providers were reluctant to use the full amount of long-acting insulin. But for long-acting insulin, endocrinologists recommended using the full-dose the day before the colonoscopy. On the day of the colonoscopy, there was a better agreement between the providers and endocrinologists. However, there was no consensus. In addition, it was important to note that there was full agreement between the two endocrinologists from two different tertiary centers.

Our study comes with the limitations of being a single-center experience with a limited number of responders (twenty-four providers). Moreover, we do not have data on hypoglycemic or hyperglycemic events on the day of colonoscopy limiting our ability to conclude if disparities in the recommendations for the management of anti-diabetic medications in the peri-colonoscopy period actually have an impact on patient outcomes. Despite the limitations, our study identifies a clinically important question that will need collaborative 
effort from professionals in the fields of endocrinology and gastroenterology to develop best practice recommendations for the management of anti-diabetic medications for type 2 DM patients undergoing colonoscopy.

Our findings indicate a marked disparity amongst gastroenterology providers (attendings, fellows, and nurse practitioners/physician assistants) with regard to endocrinologist recommendations for the management of anti-diabetic medications in the peri-colonoscopy period. It also shows a lack of familiarity of gastroenterologists with newer anti-diabetic medications. A lack of consensus can lead to confusion and mismanagement at the patient level, which can result in clinically significant hypoglycemic and hyperglycemic events. There is an unmet need for best practice recommendations for the management of anti-diabetic medications in the peri-colonoscopy period.

Conflicts of Interest

The authors have no financial conflicts of interest.

Author Contributions

Conceptualization: Byeori Lee, Deepanshu Jain

Data curation: BL, DJ

Formal analysis: BL, DJ

Supervision: Michael Rajala

Validation: MR

Writing-original draft: BL, DJ

Writing-review\&editing: MR

ORCID

Deepanshu Jain: https://orcid.org/0000-0002-0678-3515

Michael Rajala: https://orcid.org/0000-0002-2517-0175

\section{REFERENCES}

1. Seeff LC, Richards TB, Shapiro JA, et al. How many endoscopies are performed for colorectal cancer screening? Results from CDC's survey of endoscopic capacity. Gastroenterology 2004;127:1670-1677.

2. Centers for Disease Control and Prevention. National diabetes statistics report, 2017 [Internet]. Atlanta (GA): Centers for Disease Control and Prevention, US Department of Health and Human Services; c2017 [cited 2020 Mar 21]. Available from: https://dev.diabetes.org/sites/default/ files/2019-06/cdc-statistics-report-2017.pdf.

3. Geiss LS, Wang J, Cheng YJ, et al. Prevalence and incidence trends for diagnosed diabetes among adults aged 20 to 79 years, United States, 1980-2012. JAMA 2014;312:1218-1226.

4. Landreneau SW, Di Palma JA. Update on preparation for colonoscopy. Curr Gastroenterol Rep 2010;12:366-373.

5. Chokshi RV, Hovis CE, Hollander T, Early DS, Wang JS. Prevalence of missed adenomas in patients with inadequate bowel preparation on screening colonoscopy. Gastrointest Endosc 2012;75:1197-1203.

6. Hochberg I, Segol O, Shental R, Shimoni P, Eldor R. Antihyperglycemic therapy during colonoscopy preparation: a review and suggestions for practical recommendations. United European Gastroenterol J 2019;7:735-740.

7. American Diabetes Association. 15. Diabetes care in the hospital: standards of medical care in diabetes-2019. Diabetes Care 2019;42(Suppl 1):S173-S181.

8. Membership of the Working Party, Barker P, Creasey PE, et al. Peri-operative management of the surgical patient with diabetes 2015: Association of Anaesthetists of Great Britain and Ireland. Anaesthesia 2015;70:1427-1440.

9. Sudhakaran S, Surani SR. Guidelines for perioperative management of the diabetic patient. Surg Res Pract 2015;2015:284063. 